\title{
UNIFORM APPROXIMATION ON A REAL-ANALYTIC MANIFOLD
}

\author{
BY \\ MICHAEL FREEMAN $\left({ }^{1}\right)$
}

1. Introduction. Let $M$ be a compact subset of a real-analytic manifold of dimension $n$ and $F$ a set of real-analytic complex-valued functions on $M$ which separates $M$, meaning that for each pair $p, q$ of distinct points in $M$ there is a function $f$ in $F$ with $f(p) \neq f(q)$. We wish to study the Banach algebra $A$ obtained as the closure in the norm of uniform convergence on $M$ of the algebra of all polynomials in the functions of $F$ (including constants). Thus $A$ is the smallest closed subalgebra of $C(M)$ with identity which contains $F$, where $C(M)$ is all continuous complex-valued functions on $M$. Closed, separating subalgebras of $C(M)$ with identity are frequently called function algebras, and the term is used elsewhere in much more general circumstances, where arbitrary compact Hausdorff spaces are admitted for $M$.

As we note below, the study of this type of function algebra includes the classical problem of uniform polynomial approximation on certain nolynomially convex subsets of complex Euclidean space $\boldsymbol{C}^{n}$.

Our study of these algebras continues a program initiated by Wermer [12], [13], and treated by the author [2], Wells [11], Nirenberg and Wells [9], [10], and very recently by Hörmander and Wermer [6]. In all of this work it has been shown that the set

$E=\left\{p \in M: d f_{1} \wedge \cdots \wedge d f_{n}(p)=0\right.$ for all $n$-tuples $\left\{f_{1}, \ldots, f_{n}\right\}$ of functions in $\left.F\right\}$ plays a major role in determining the structure of $A$. Our principal object in this paper is to prove a result announced earlier [3].

THeORem 1. If $M_{A}=M$ then $A$ contains the ideal of all continuous complex-valued functions which vanish on $E$.

Here $M_{A}$ is the spectrum or maximal ideal space of the Banach algebra $A$, and consists of all algebra homomorphisms of $A$ onto $C$. Each point $p$ of $M$ provides such a homomorphism, defined by sending a function $f$ in $A$ into $f(p)$. The hypothesis $M_{A}=M$ means that all homomorphisms arise in this manner. It is a necessary condition for the conclusion when $E$ is empty and in certain other cases [2]. We refer to [2] for further properties of $E$.

Received by the editors May 23, 1968 and, in revised form, February 17, 1969.

(1) This work was supported in part by Army Grant DA-ARO-D-31-124-G866 while the author was at Brandeis University. 
The theorem says nothing about the behavior of $A$ on $E$, a problem of great interest since the theorem shows it equivalent to the problem of describing $A$ (it is easy to see that a continuous function $f$ on $M$ is in $A$ if and only if its restriction to $E$ coincides with that of some function in $A$ ). In the case where $M$ is contained in a real-analytic submanifold of $C^{n}$, and with the usual coordinate functions comprising $F, A$ is the algebra of all continuous functions on $M$ which can be approximated uniformly by polynomials. Here the condition $M_{A}=M$ is equivalent to the assertion that $M$ is polynomially convex [5], [14]. In this case Hörmander and Wermer [6], and earlier Wermer [13] in a special case, have shown that $A$ is the set of all continuous functions on $M$ which admit uniform approximation on $E$ by functions holomorphic in a neighborhood of $E$. Their result of course contains Theorem 1 in this case. Wermer [13] also gives a differential description of $A$ in a special case. A description of this type is conjectured in [3] for the more general situation treated here, but we have not proved it.

The author proved Theorem 1 when $n=2$ in [2], by extending techniques first used by Wermer [13]. The same basic ideas are used here, but a number of modifications and extensions have been necessary to adapt the earlier proof to manifolds of dimension greater than two.

A simple example which verifies Theorem 1 is obtained when

$$
M=\left\{(x, y, t) \in R^{3}: x^{2}+y^{2}+t^{2} \leqq 1\right\}
$$

is the closed unit ball in real Euclidean space $\boldsymbol{R}^{3}$ and $F=\{f, g, h\}$ where

$$
f(x, y, t)=x+i y=z, g(x, y, t)=t \bar{z} \text {, and } h(x, y, t)=t .
$$

Then the function $(f, g, h): M \rightarrow C^{3}$ with the indicated coordinates maps $M$ homeomorphically onto a compact polynomially convex [4], [5] subset of $\boldsymbol{C}^{3}$, so [5] $M_{A}=M$. Clearly, $E=\{(x, y, t): t=0\}$. Moreover, $A$ contains $f h, g$, and $h$, which together separate $M-E$, which have no common zero there, and which generate an algebra closed under complex conjugation. An application of the Stone-Weierstrass theorem now shows that $A$ contains every continuous function which vanishes on $E$.

2. Proof of Theorem 1. The argument is similar and in some places identical to that used before [2]. We show again that every bounded regular complex Borel measure which annihilates $A$ also annihilates every continuous function which vanishes on $E$. This follows from Theorem 2 below in exactly the same way as it did in [2]. Theorem 2 reduces the study of such a measure $\mu$ to the study of the family of bounded, regular, compactly supported Borel measures $f_{*} \mu$ induced on the plane from $\mu$ by certain functions in $A$. These measures are defined for each Borel set $E$ by $f_{*} \mu(E)=\mu\left(f^{-1}(E)\right)$ and their relevant elementary properties are listed in [2].

We write $\mu \perp f$ if $\int f d \mu=0$ and write $\mu \perp A$ if this holds for all functions in $A$. If $f_{1}, \ldots, f_{k}$ are functions on $M$ we denote by

$$
\left(f_{1}, \ldots, f_{k}\right): M \rightarrow C^{k}
$$

the map with these functions as coordinates. 
The following well-known facts are collected as lemmas for future use.

LEMMA 1. If $\nu$ is a bounded regular complex Borel measure with compact support in $C$, then

(1) $\int d|\nu|(\lambda) /|\lambda-z|$ is finite for almost all $z$ in $C$ (in the sense of Lebesgue measure), and

(2) if $K$ is compact in $C$ and $\int d \nu(\lambda) /(\lambda-z)=0$ for almost all $z$ in $C-K$ (in the same sense), then support $\nu \subset K$.

In particular, if (1) holds for almost all $z$ in $C$, then it follows from (2) that $\nu=0$.

For a proof, the reader is referred to [14].

LEMMA 2. If $M$ is a compact subset of a complex manifold and $G$ is a holomorphic map of a neighborhood of $M$ into $C^{n}$ which is injective and nonsingular on $M$, then $G$ is injective and nonsingular on some neighborhood of $M$.

LEMMA 3. Let $X$ be a compact Hausdorff space and $F$ a subset of $C(X)$ satisfying

(3) $F$ separates $X$, and

(4) for each $x$ in $X$ there exists a finite subset of $F$ which separates some neighborhood of $x$.

Then there exists a finite subset of $F$ which separates $X$.

This type of result has been used by Narasimhan [8], aind was brought to my attention by $\mathrm{H}$. Rossi.

Proof. Property (4) and compactness yield open sets $U_{1}, \ldots, U_{k}$ and a finite subset $\left\{g_{1}, \ldots, g_{l}\right\}$ of $F$ which separates each $U_{i}$. Property (3) and the compactness of $X \times X$ provide open sets $V_{1}, \ldots, V_{p}, W_{1}, \ldots, W_{p}$ and functions $g_{l+1}, \ldots, g_{l+p}$ in $F$ such that

$$
X \times X-\bigcup_{i=1}^{k} U_{i} \times U_{i} \subset \bigcup_{i=1}^{p} V_{i} \times W_{i}
$$

and

$$
g_{l+j}\left(V_{j}\right) \cap g_{l+j}\left(W_{j}\right)=\varnothing, \quad j=1, \ldots, p .
$$

It follows easily that $\left\{g_{1}, \ldots, g_{l+p}\right\}$ separates $X$.

LEMMA 4. Let $M$ be a compact Hausdorff space and $F$ a separating subset of $C(M)$. If $U$ is open in $M$ and $\left\{f_{1}, \ldots, f_{k}\right\}$ is a subset of $F$ which separates $U$, then for each compact subset $K$ of $U$ there exist functions $f_{k+1}, \ldots, f_{m}$ in $F$ such that $\left(f_{1}, \ldots, f_{m}\right)(K)$ is disjoint from $\left(f_{1}, \ldots, f_{m}\right)(M-K)$.

Proof. Since $F$ separates $M$, a standard compactness argument shows that there exist functions $f_{k+1}, \ldots, f_{m}$ in $F$ such that

$$
\left(f_{k+1}, \ldots, f_{m}\right)(K) \text { is disjoint from }\left(f_{k+1}, \ldots, f_{m}\right)(M-U) .
$$

It is straightforward to verify that $\left\{f_{1}, \ldots, f_{m}\right)$ has the stated property. 
THEOREM 2. If $M_{A}=M, \mu \perp A$, and $f$ is a polynomial in the functions of $F$, then

$$
\int_{C} \frac{d\left(f_{*} \mu\right)(\lambda)}{\lambda-a}=0
$$

for almost all points a in $C-f(E)$. Thus by Lemma 1, support $f_{*} \subset \subset f(E)$.

Proof. As in [2], it will suffice to show that $\int d \mu /(f-a)=0$ for all points $a$ in $C-f(E)$ for which the integral is absolutely convergent. For each such $a$, we construct a sequence $\left\{f_{n}\right\}$ of functions in $A$ such that

$$
f_{n} \rightarrow 1 /(f-a) \text { a.e. }|\mu| \text {, and }\left|f_{n}\right| \leqq 2 /|f-a| \text { a.e. }|\mu| \text {. }
$$

These functions are obtained as before from the solution of a certain Cousin Problem I on a domain in $\boldsymbol{C}^{n}$. This problem has the same basic structure as it did in [2], but is somewhat more complicated because of the higher dimension. To set it up, we appeal to a result of Whitney and Bruhat [15], which states that there exists a complex manifold $\tilde{M}$ in which the ambient manifold of $M$ can be imbedded as a real-analytic submanifold in such a way that every real-analytic function on $M$ can be extended to a holomorphic function on an $\tilde{M}$-neighborhood of $M$. While some of the constructions below could be executed on $\tilde{M}$, it is more convenient to transfer immediately to complex Euclidean space. The basic idea of the proof is clearest when $F$ is finite, so we present that case first. Technical modifications required to handle the general situation are given afterwards.

Case of finite $F$. Here the functions in $F$ comprise the coordinates of a map

$$
H=\left(f_{1}, \ldots, f_{p}\right): M \rightarrow C^{p} .
$$

By our assumption, $f=q \circ H$ for some polynomial $q$ in $p$ variables. Given a point $a$ in $C-f(E)$ we claim that:

There exists an open set $W \supset H(M) \cap q^{-1}(a)$ and a function $k$ holomorphic on $W$ such that $k\left|H(M) \cap W=(q-a)^{*}\right| H(M) \cap W$. Here the * denotes complex conjugation.

There is an $\tilde{M}$-open set $\tilde{V}$ which contains $f^{-1}(a)$ and to which $H$ has a holomorphic extension $\tilde{H}=\left(f_{1}, \ldots, \tilde{f}_{p}\right)$. Since $a$ is not in $f(E)$ the $p$-form $d f_{1} \wedge \cdots \wedge d f_{p}$ has no zeros on $f^{-1}(a)$ so the same is true of $d \tilde{f}_{1} \wedge \cdots \wedge d \tilde{f}_{p}$. Since $\tilde{H}$ is injective on $M$ we can use Lemma 2 to choose $\tilde{V}$ small enough so that $\tilde{H}$ imbeds $\tilde{V}$ as a complex submanifold $V$ of $C^{p}$.

We may assume that $(f-a)^{*}$ has a holomorphic extension to $\tilde{V}$. Now $V$ is a closed submanifold of some open set $U$ in $C^{p}$ (for instance, a union of ambient coordinate neighborhoods whose slices define $V$ locally), and since $H(M \cap \tilde{V})$ is disjoint from $H(M-\tilde{V})$ we can remove the compact set $H(M-\tilde{V})$ from $U$ to obtain $V$ as a closed submanifold of the open set $U$ and satisfying

$$
U \cap H(M)=V \cap H(M)=H(M \cap \tilde{V}) .
$$


The set $H(M) \cap q^{-1}(a)$ is polynomially convex [5], as a consequence [14] of our assumption that $M_{A}=M$. Therefore there exists [5] a domain of holomorphy $W$ such that $H(M) \cap q^{-1}(a) \subset W \subset U$. We have an extension of $(f-a)^{*}=(q-a)^{*} \circ H$ to a holomorphic function on $\tilde{V}$. Because of (5), composition of this extension with $(\tilde{H} \mid \tilde{V})^{-1}: V \rightarrow \tilde{V}$ yields a holomorphic extension to $V$ of $(q-a)^{*} \mid H(M) \cap V$. The theorem of Grauert and Docquier [4, Theorem 8, pp. 257-258], which says that $W \cap V$ is a holomorphic retraction of $W$, finally yields the desired extension $k$ on $W$.

We wish now to proceed along lines similar to [2], and construct functions $h$ and $h_{1}$ in $A$ such that $h=(f-a) h_{1}$ and $h(M) \subset\{w:|w-1|>1\} \cup\{0\}$. A "local" solution to this problem is given by $h_{1}=-k \circ H$ and $h=(f-a) h_{1}$. However $k \circ H$ is not in $A$, and we wish to use $k$ to obtain a holomorphic function $\psi$ with appropriate divisibility properties in a neighborhood of $H(M)$. Since $H(M)$ is polynomially convex, the Oka-Weil theorem [5, Theorem 2.7.7, p. 55] will imply that $h=-\psi \circ H$ is in $A$. Let $g=(q-a) k$, a function holomorphic on $W$.

Then there exists a function $\psi$ holomorphic on a neighborhood of $H(M)$ such that

(6) $\psi$ has no zeros on $H(M)-q^{-1}(a)$, and

(7) $\psi$ is divisible by $g$ in a neighborhood of $H(M) \cap q^{-1}(a)$ and the holomorphic quotient $\psi / g$ has the value 1 everywhere on $H(M) \cap q^{-1}(a)$.

This function $\psi$ is exhibited as the solution to a Cousin Problem I [5] with data determined from $g$ as follows. Since $g|H(M) \cap W=| q-\left.a\right|^{2} \mid H(M) \cap W$, it follows that

$$
H(M) \cap\{\operatorname{Re} g=0\}=H(M) \cap q^{-1}(a) .
$$

Therefore by shrinking $W$ if necessary, it can be assumed that $\{\operatorname{Re} g=0\}^{-}$is disjoint from $H(M)-W$ (here the superscript bar denotes closure).

Thus $C^{p}-q^{-1}(a)-\{\operatorname{Re} g=0\}^{-}$is an open set containing $H(M)-W$, so that $W$ and this set constitute an open cover of $H(M)$. Since $H(M)$ is polynomially convex, there exists an open domain of holomorphy $S$ in $C^{p}$ such that

$$
H(M) \subset S \subset W \cup\left[C^{p}-q^{-1}(a)-\{\operatorname{Re} g=0\}^{-}\right] .
$$

Replacing $W$ by its intersection with $S$, we have $W \subset S$. Since $\{\operatorname{Re} g=0\}$ is closed in $S$, the set $T=S-q^{-1}(a)-\{\operatorname{Re} g=0\}$ is open and $S=W \cup T$.

We have designed $W, T$, and $g$ so that $g$ has a holomorphic logarithm $\log g$ on

$$
W \cap T=W-q^{-1}(a)-\{\operatorname{Re} g=0\},
$$

and so that $(\log g) /(q-a)$ is holomorphic on $W \cap T$. Therefore the Cousin Problem I defined on $S$ for the covering $\{W, T\}$ by $(\log g) /(q-a)$ has a solution [5]; that is, there exist functions $g_{1}$ holomorphic on $T$ and $g_{2}$ holomorphic on $W$ such that

$$
g_{1}-g_{2}=(\log g) /(q-a) \text { on } W \cap T .
$$

Thus $\log g+(q-a) g_{2}=(q-a) g_{1}$ on $W \cap T$ so the holomorphic functions

$$
g \exp \left((q-a) g_{2}\right) \text { on } W \text { and } \exp \left((q-a) g_{1}\right) \text { on } T
$$


coincide on $W \cap T$. Hence they define a holomorphic function $\psi$ on $S$ with the desired properties.

This result is used to construct functions $h$ and $h_{1}$ in $A$ such that

(8) $h$ has no zeros on $H(M)-q^{-1}(a)$,

(9) $h=(f-a) h_{1}$, and

(10) $h(M) \subset\{w:|w-1|>1\} \cup\{0\}$.

From (6) and (7) it follows that $\psi_{1}=\psi /(q-a)$ is holomorphic in a neighborhood of $H(M)$. By (7) there exists an $H(M)$-neighborhood $P$ of $H(M) \cap q^{-1}(a)$ on which $\operatorname{Re}(\psi / g)>0$. It follows from this and the positivity of $g$ on $H(M)-q^{-1}(a)$ that $\operatorname{Re} \psi>0$ on $P-q^{-1}(a)$. The function $\psi$ has no zeros on the compact set $H(M)-P$, so after multiplication of $\psi$ by a suitable positive constant, it will satisfy $|\psi| \geqq 2$ on $H(M)-P$. We have already noted that $h=-\psi \circ H$ and $h_{1}=-\psi_{1} \circ H$ are in $A$, and they clearly have the properties (8), (9) and (10).

These functions are used exactly as in $\left[2\right.$, p. 54] to construct the sequence $\left\{f_{n}\right\}$. There we defined the rational functions $\phi_{n}$ by

$$
\phi_{n}(w)=\frac{1}{w}\left(1-\frac{1}{(w-1)^{2 n}}\right), \quad n=1,2, \ldots
$$

and showed easily that the sequence $\left\{f_{n}\right\}$ of functions in $A$ defined by $f_{n}=\left(\phi_{n} \circ h\right) h_{1}$, $n=1,2, \ldots$ has the properties set forth at the beginning of the proof. Theorem 2 is thereby proved when $F$ is finite.

Proof of Theorem 2 for arbitrary $F$. The proof has the same basic structure as before, but it must surmount two additional difficulties. Since we cannot expect to find any finite subset of $F$ to provide the coordinates of a homeomorphism of $M$ into a complex Euclidean space, the separation arguments made at the beginning are somewhat more involved. A more serious problem is that the image of $M$ under a map $G=\left(f_{1}, \ldots, f_{m}\right)$ with the $f_{j}$ 's in $F$ will not necessarily be polynomially convex. Because of this, domains of holomorphy corresponding to $W$ and $S$ will be harder to find. To construct them, we will use a standard technique due to Arens and Calderón [1], [5].

We again choose a point $a$ in $C-f(E)$ and claim that there exist functions $\left\{f_{1}, \ldots, f_{m}\right\}$ in $F$, an open set $U$ in $C^{m}$, and a closed complex submanifold $V$ of $U$ such that if $G=\left(f_{1}, \ldots, f_{m}\right)$ then

(11) $f=q \circ G$ for some polynomial $q$ in $m$ variables,

(12) $V \supset G(M) \cap q^{-1}(a)$,

(13) $U \cap G(M)=V \cap G(M)=G(M \cap \tilde{V})$, and

(14) $(q-a)^{*}$ extends from $G(M) \cap V$ to a function $k$ holomorphic on $V$.

To begin the construction of $G, U$, and $V$ we note that for each point of $f^{-1}(a)$ there can be found functions $f_{1}, \ldots, f_{n}$ in $F$ and a neighborhood of the point on which $d f_{1} \wedge \cdots \wedge d f_{n}$ has no zeros. These functions extend to holomorphic functions $\tilde{f}_{1}, \ldots, \tilde{f}_{n}$ on an $\tilde{M}$-neighborhood of the point on which the extended form $d \tilde{f}_{1} \wedge \cdots \wedge d \tilde{f}_{n}$ has no zeros. Since $f^{-1}(a)$ is compact it can be covered by finitely 
many such neighborhoods with the result that there exist functions $f_{1}, \ldots, f_{l}$ in $F$ whose holomorphic extensions provide a map $\left(\tilde{f}_{1}, \ldots, \tilde{f}_{l}\right)$ with maximum rank $n$ on some $\tilde{M}$-neighborhood of $f^{-1}(a)$. Since $F$ separates $M$, Lemma 3 applied to $F$ and $f^{-1}(a)$ yields the existence of functions $f_{l+1}, \ldots, f_{m}$ in $F$ whose adjunction provides a map

$$
G=\left(f_{1}, \ldots, f_{m}\right): M \rightarrow C^{m}
$$

which is injective and of rank $n$ in an $M$-neighborhood of $f^{-1}(a)$. By Lemma 2, the map $\left(\tilde{f}_{1}, \ldots, \tilde{f}_{m}\right)$ imbeds an open $\tilde{M}$-neighborhood $\tilde{V}$ of $f^{-1}(a)$ as a complex submanifold $V$ of $C^{m}$. This property is clearly unaffected by the adjunction of more coordinate functions, so we may assume that (11) is true. Statement (12) is then immediate.

By applying Lemma 4 to $M$ and $M \cap \tilde{V}$ and passing from $\tilde{V}$ to a relatively compact subset of it which contains $f^{-1}(a)$, we can assume that $G(M \cap \tilde{V})$ and $G(M-\tilde{V})$ are disjoint. Just as before we can arrange that $V$ is a closed submanifold of an open set $U$ in $C^{m}$ which satisfies (13), and find an extension $k$ verifying (14).

However, $U$ need not contain a domain of holomorphy containing $G(M) \cap q^{-1}(a)$, since the latter set is not necessarily polynomially convex. Hence the theorem of Grauert and Docquier cannot yet be used to extend $k$ to an open set in $\boldsymbol{C}^{m}$.

To effect this extension and thus prepare the way for the Cousin I construction above, we shall use a technique of Arens and Calderón [1], [5]. In fact, we assert that there are functions $f_{m+1}, \ldots, f_{p}$ in $F$ such that if $H=\left(f_{1}, \ldots, f_{p}\right)$ and $B$ is the closed subalgebra of $A$ with identity generated by $\left\{f_{1}, \ldots, f_{p}\right\}$, then there exists $a$ domain of holomorphy $W$ in $C^{p}$ such that $M_{B} \cap q^{-1}(a) \subset W \subset U \times C^{p-m}$ and $k$ extends to a holomorphic function on $W$. Here we have made the usual identification of the maximal ideal space $M_{B}$ of $B$ with the polynomially convex hull of $H(M)$ in $C^{p}$, and the functions $k$ and $q$ are transferred in the obvious way to functions on $V \times C^{p-m}$ and $C^{p}$, respectively.

These additional functions are obtained by means of the Lemma of Arens and Calderón [5], which says that there can be found $f_{m+1}, \ldots, f_{p}$ in $F$ such that with $H$ and $B$ as defined above and $\sigma_{B}\left(f_{1}, \ldots, f_{m}\right)$ the joint spectrum [5] of the indicated functions relative to $B$, we have

$$
\sigma_{B}\left(f_{1}, \ldots, f_{m}\right) \subset U \cup\left[C^{m}-q^{-1}(a)\right] .
$$

Their lemma is applicable because $U \supset G(M) \cap q^{-1}(a)$, so the right side of (15) is an open neighborhood of $G(M)$.

Now since $\sigma_{B}\left(f_{1}, \ldots, f_{m}\right)$ is the projection of $M_{B}$ on $C^{m}$, we have

$$
M_{B} \subset\left[U \times C^{p-m}\right] \cup\left[C^{p}-q^{-1}(a)\right], \quad \text { so } M_{B} \cap q^{-1}(a) \subset U \times C^{p-m} .
$$

Moreover, $M_{B} \cap q^{-1}(a)$ is polynomially convex, since $M_{B}$ has this property. Thus there exists a domain of holomorphy $W$ in $C^{p}$ with

$$
M_{B} \cap q^{-1}(a) \subset W \subset U \times C^{p-m} .
$$


Finally, $\left(V \times C^{p-m}\right) \cap W$ is a closed submanifold of $W$, and the function $k$ extends to $W$ exactly as it did before.

We can now construct a function $\psi$ holomorphic in a neighborhood of $M_{B}$ with no zeros on $M_{B}-q^{-1}(a)$, divisible by $g=(p-a) k$ in a neighborhood of $M_{B} \cap q^{-1}(a)$ and such that the holomorphic quotient $\psi / g$ has the value 1 everywhere on $H(M) \cap q^{-1}(a)$.

For we have again that

$$
\{\operatorname{Re} g=0\} \cap H(M)=q^{-1}(a) \cap H(M),
$$

and we may therefore assume that $\{\operatorname{Re} g=0\}^{-}$is disjoint from $H(M)-W$. However, it may not be the case that $\{\operatorname{Re} g=0\}^{-}$is disjoint from $M_{B}-W$. If not, this separation may be achieved by another application of the Arens-Calderón Lemma, noting that

$$
W \cup\left[C^{p}-q^{-1}(a)-\{\operatorname{Re} g=0\}^{-}\right]
$$

is an open neighborhood of $H(M)$ and proceeding as above. In other words, we can assume that

$$
M_{B} \subset W \cup\left[C^{p}-q^{-1}(a)-\{\operatorname{Re} g=0\}^{-}\right],
$$

which enables the construction of $\psi$ as the solution to the same Cousin Problem I that we have already described. The proof is then completed exactly as it was when $F$ is finite.

3. Some conditions for $A=C(M)$.

Corollary 1. If $M_{A}=M$ and $E$ is totally disconnected, then $A=C(M)$.

This result appears in [2]. Since it depends solely on the conclusion of Theorem 1 and not on the dimension of the ambient manifold of $M$, the proof given there holds without modification.

In [2] we also deduced for the two-dimensional case that $A=C(M)$ if $M_{A}=M$ and $E$ has Lebesgue measure zero. The example presented in $\S 1$ shows that this result fails in higher dimensions. In this example, $E$ has three-dimensional Lebesgue measure zero but every function in $A$ is a uniform limit on $E$ of polynomials in $f$, with $f(x, y, t)=x+i y$. Thus each function in $A$ is holomorphic on $E$ (in the obvious sense), so that $A \neq C(M)$. However, a stronger measure-theoretic restriction on $E$ will still yield the same result:

Corollary 2. If $M_{A}=M$ and $E$ has two-dimensional Hausdorff measure zero, then $A=C(M)$.

Proof. It is easily seen that the image by a continuously differentiable map of a set of two-dimensional Hausdorff measure zero also has this property. Because of the relation [7, p. 104] between Hausdorff two-dimensional measure and plane Lebesgue measure, we then have for any polynomial $f$ in the functions of $F$ that 
$f(E)$ has measure zero. From Theorem 2 it follows that $f_{*} \mu=0$, which implies that $\mu=0[2$, p. 56].

We remark that it is clear how, by adjoining more coordinates, an example of the type presented in $\S 1$ can be constructed where $M$ has any dimension greater than two but $E$ has Hausdorff three-dimensional measure zero and $A \neq C(M)$.

\section{REFERENCES}

1. R. Arens and A. P. Calderón, Analytic functions of several Banach algebra elements, Ann. of Math. 62 (1955), 204-216.

2. M. Freeman, "Some conditions for uniform approximation on a manifold" in Function algebras, Scott, Foresman and Co., Chicago, Ill., 1966.

3. - - Function algebras on a manifold, Proc. Rice Univ. Conf. on Complex Analysis, April 1967, Rice Univ. Studies 54 (1968), 15-19.

4. R. Gunning and H. Rossi, Analytic functions of several complex variables, Prentice-Hall, Englewood Cliffs, N. J., 1965.

5. L. Hörmander, An introduction to complex analysis in several variables, Van Nostrand, Princeton, N. J., 1966.

6. L. Hörmander and J. Wermer, Uniform approximation on compact sets in $C^{n}$, Math. Scand. (to appear).

7. W. Hurewicz and H. Wallman, Dimension theory, Princeton Univ. Press, Princeton, N. J., 1941.

8. R. Narasimhan, Imbedding of holomorphically complete complex spaces, Amer. J. Math. 82 (1960), 917-934.

9. R. Nirenberg and R. O. Wells, Jr., Holomorphic approximation on real submanifolds of a complex manifold, Bull. Amer. Math. Soc. 73 (1967), 378-381. (This is an announcement of some results in reference [10].)

10. - - Approximation theorems on differentiable submanifolds of a complex manifold, Trans. Amer. Math. Soc. 142 (1969), 15-35.

11. R. O. Wells, Jr., Holomorphic approximation on real-analytic submanifolds of a complex manifold, Proc. Amer. Math. Soc. 17 (1966), 1272-1275.

12. J. Wermer, Approximation on a disk, Math. Ann. 155 (1964), 331-333.

13. - Polynomially convex disks, Math. Ann. 158 (1965), 6-10.

14. —_ Banach algebras and analytic functions, Advances in Math. 1 (1961), 51-102.

15. H. Whitney and F. Bruhat, Quelques propriétés fondamentales des ensembles analytiquesréeles, Comment. Math. Helv. 33 (1959), 132-160.

RICE UNIVERSITY,

Houston, TeXas 